\title{
Early allograft dysfunction after liver transplantation with donation after cardiac death donors
}

\author{
Junbin Zhou ${ }^{1,2}$, Qiang Wei ${ }^{1,2}$, Shusen Zheng ${ }^{1,2}$, Xiao Xu ${ }^{1,2}$ \\ ${ }^{1}$ Department of Hepatobiliary and Pancreatic Surgery, First Affiliated Hospital, Zhejiang University School of Medicine, Hangzhou 310003, China; \\ ${ }^{2}$ NHFPC Key Laboratory of Combined Multi-Organ Transplantation, Hangzhou 310003, China \\ Correspondence to: Prof. Xiao Xu, MD, PhD. Department of Hepatobiliary and Pancreatic Surgery, First Affiliated Hospital, Zhejiang University \\ School of Medicine, 79 Qingchun Road, Hangzhou 310003, China. Email: zjxu@zju.edu.cn.
}

Submitted May 16, 2019. Accepted for publication Jun 13, 2019.

doi: 10.21037/hbsn.2019.07.07

View this article at: http://dx.doi.org/10.21037/hbsn.2019.07.07

Liver transplantation (LT) is recognized as one of the effective approaches for end-stage liver diseases, and early allograft dysfunction (EAD) is a frequent complication after LT, which is always associated with higher morbidity and mortality.

Since 2015, the donation after cardiac death (DCD) from citizen-donors has been the sole legal source of donor organs in China. This also increases the proportion of marginal donors such as steatosis, senile donor liver, infection, or unknown cause of death. Marginal donor liver enlarges the source of donor's liver but increases the incidence of EAD after transplant. The incidence of EAD after LT ranges from $20 \%$ to $40 \%$ (1). Olthoff and colleagues (2) defined EAD as the presence at least one of the following criteria: bilirubin $\geq 10 \mathrm{mg} / \mathrm{dL}$ on postoperative day 7; international normalized ratio (INR) $\geq 1.6$ on postoperative day 7; alanine aminotransferase (ALT) or aspartate aminotransferase (AST) $>2,000$ IU/L within the first postoperative 7 days. Though as the most widely used definition currently, it is a static definition and not taking the rate of changing variables into account $(1,3)$. Recently, the rate of normalization of bilirubin levels and serum AST was validated to be associated with $\mathrm{EAD}$, and a liver graft assessment following transplantation (L-GrAFT) model was constructed to predict 3-month graft failure, which was the first dynamic model considering the rate of changing variables (1). In addition, the L-GrAFT model showed superior discrimination when compared with Olthoff's definition by the area under the curve measurement (AUC: 0.85 vs. 0.68). In general, a uniform definition of acceptance is needed, which will be beneficial for multicenter cooperation and promoting the research on EAD.
The risk factors of EAD can be mainly divided into three categories: the donor, the recipient, and surgeryrelated factors. Donor-related factors include body mass index, age, and sex; in addition, donor liver apoptosis and steatosis were also reported to be responsible for graft dysfunction $(4,5)$, but another study (6) indicated that donor liver microvesicular steatosis is not related to EAD, which requires further confirmation. Considering recipientrelated factors, recent studies $(7,8)$ showed that male recipients who have hepatocellular carcinoma, hepatitis $\mathrm{C}$ virus infection, pre-transplant diabetes, post-transplant biliary complications, and lower cholesterol are more likely to develop EAD. As for surgery-related factors, prolonged cold ischemic time, warm ischemic time, and operative time contribute to EAD. The donor liver biology plays an important role in the occurrence and development of EAD. While risk factors have been widely studied, there is a lack of research on the molecular mechanism of EAD. Study on molecular mechanism will help to understand EAD in-depth; for example, a group studied the genomic profiling of EAD grafts and found some pathways (PPAR $\alpha$ and NF- $\mathrm{BB}$ ) are relevant (9). Generally, because EAD is a multifactorial complication post-LT, many studies $(7,8)$ have been done to find controllable risk factors to achieve more efficient prediction and prevention of $\mathrm{EAD}$, through which the patient and graft survival can be improved.

Predicting the occurrence of EAD more accurately can help to prevent and take earlier therapeutic methods. Firstly, serum factor $\mathrm{V}$ measured on postoperative day one was used as an earlier biomarker of EAD for the first time in 2018 (3). Secondly, donor liver apoptosis was found to be related to $\mathrm{EAD}$, and a nomogram was built on apoptosis and five 
other predictors (AUC: 0.847) (4). Thirdly, metabolomics method is increasingly becoming a useful tool for assessing graft functions, especially for DCD grafts, through which lactate was found to be an efficient predictor for $\mathrm{EAD}$ (AUC: 0.906) (10). Despite that many metabolomics methodologies are not used clinically due to complicated sample handling steps, $1 \mathrm{H}$ high-resolution magic-angle-spinning nuclear magnetic resonance (1H HR-MAS NMR) spectroscopy has provided an effective solution and has been applied in LT, which will help to predict EAD more accurately.

Given that EAD is always associated with high mortality unless graft function is restored or re-transplantation, there is a need to develop prevention and treatments for dysfunction liver support. Firstly, as for prevention techniques, preservation of donors is noteworthy. DCD donors are usually suffering ischemia injury due to circulatory arrest, which may contribute to a prolonged warm ischemic time and lead to a higher incidence of EAD. Thus, when comparing with static cold storage which is the current standard method for liver preservation, normothermic regional perfusion is a newly-developed method with many advantages such as improving the quality of DCD grafts and making graft function assessment convenient. Moreover, normothermic regional perfusion has already been used in LT with DCD grafts (11). Secondly, considering the therapies, therapeutic plasma exchange was applied in EAD patients recently (12). By removing toxic substances (i.e., plasma bilirubin) and supplying coagulation factors which are absent in EAD patients, therapeutic plasma exchange is efficient in reducing bilirubin levels and INR, and improving recipients and grafts survival.

In conclusion, firstly, many studies on $\mathrm{EAD}$ were based on a single transplant center which cannot be generalized. Thus, the collaboration of multicenter is needed for further research, which requires a more uniform, efficient, and widely accepted definition. Secondly, as DCD donors are associated with a higher risk of $\mathrm{EAD}$, more certain risk factors or biomarkers must be studied to refine current predicting models which will help to match donors and recipients, predict the occurrence of $\mathrm{EAD}$, take earlier therapies and finally improve the prognosis post-LT. Moreover, metabolomics profiling is becoming a powerful tool for evaluating graft functions post-LT. Thirdly, based on the controllable risk factors founded, novel technologies should be developed to reduce the risk of EAD, such as normothermic regional perfusion. Fourthly, more effective therapies should be developed to support EAD recipients until the grafts are functional or re-transplantation. Finally, because little research has been reported on the molecular mechanism of EAD, which is necessary for an in-depth understanding, more attention should be paid to this area in the future.

\section{Acknowledgments}

Funding: This study was supported by grants from the National Natural Science Foundation of China (81570589 and 81800578), the National Science and Technology Major Project (2017ZX10203205).

\section{Footnote}

Conflicts of Interest: The authors have no conflicts of interest to declare.

Ethical Statement: The authors are accountable for all aspects of the work in ensuring that questions related to the accuracy or integrity of any part of the work are appropriately investigated and resolved.

\section{References}

1. Agopian VG, Harlander-Locke MP, Markovic D, et al. Evaluation of Early Allograft Function Using the Liver Graft Assessment Following Transplantation Risk Score Model. JAMA Surg 2018;153:436-44.

2. Olthoff KM, Kulik L, Samstein B, et al. Validation of a current definition of early allograft dysfunction in liver transplant recipients and analysis of risk factors. Liver Transpl 2010;16:943-9.

3. Gorgen A, Prediger C, Prediger JE, et al. Serum Factor $\mathrm{V}$ is a Continuous Biomarker of Graft Dysfunction and a Predictor of Graft Loss After Liver Transplantation. Transplantation 2019;103:944-51.

4. Zhu Z, Tang Y, Huang S, et al. Donor liver apoptosis is associated with early allograft dysfunction and decreased short-term graft survival after liver transplantation. Clin Transplant 2018;32:e13438.

5. Linares I, Hamar M, Selzner N, et al. Steatosis in Liver Transplantation: Current Limitations and Future Strategies. Transplantation 2019;103:78-90.

6. Andert A, Ulmer TF, Schoning W, et al. Grade of donor liver microvesicular steatosis does not affect the postoperative outcome after liver transplantation. Hepatobiliary Pancreat Dis Int 2017;16:617-23.

7. Huesing-Kabar A, Dohna CZ, Heinzow H, et al. Risk 
factors for allograft failure in liver transplant recipients. $Z$ Gastroenterol 2018;56:745-51.

8. Yang J, Wang HQ, Yang JY, et al. Role of the postoperative cholesterol in early allograft dysfunction and survival after living donor liver transplantation. Hepatobiliary Pancreat Dis Int 2017;16:610-6.

9. Kurian SM, Fouraschen SM, Langfelder P, et al. Genomic profiles and predictors of early allograft dysfunction after human liver transplantation. Am J Transplant 2015;15:1605-14.

Cite this article as: Zhou J, Wei Q, Zheng S, Xu X. Early allograft dysfunction after liver transplantation with donation after cardiac death donors. HepatoBiliary Surg Nutr 2019;8(5):566-568. doi: 10.21037/hbsn.2019.07.07
10. Faitot F, Besch C, Battini S, et al. Impact of real-time metabolomics in liver transplantation: Graft evaluation and donor-recipient matching. J Hepatol 2018;68:699-706.

11. De Carlis R, Di Sandro S, Lauterio A, et al. Successful donation after cardiac death liver transplants with prolonged warm ischemia time using normothermic regional perfusion. Liver Transpl 2017;23:166-73.

12. Choe W, Kwon SW, Kim SS, et al. Effects of therapeutic plasma exchange on early allograft dysfunction after liver transplantation. J Clin Apher 2017;32:147-53. 